\title{
Levinson theorem in two dimensions
}

\author{
Qiong-gui Lin \\ China Center of Advanced Science and Technology (World Laboratory), \\ P.O.Box 8730, Beijing 100080, P.R.China \\ and \\ Department of Physics, Zhongshan University, Guangzhou 510275, P.R.China 円
}

\begin{abstract}
A two-dimensional analogue of Levinson's theorem for nonrelativistic quantum mechanics is established, which relates the phase shift at threshold(zero momentum) for the $m$ th partial wave to the total number of bound states with angular momentum $m \hbar(m=0,1,2, \ldots)$ in an attractive central field.
\end{abstract}

PACS number(s): 34.10.+x, 34.90.+q, 03.65.-w

\footnotetext{
${ }^{1}$ Mailing Address
} 


\section{$\S 1$ Introduction}

In 1949, a theorem in quantum mechanics was established by Levinson[1], which relates the phase shift at threshold(zero momentum) for the $l$ th partial wave, $\delta_{l}(0)$, to the number of bound states with the same azimuthal quantum number, $n_{l}$. This is one of the most interesting and beautiful results in nonrelativistic quantum theory. The subject was then studied by many authors, some are listed in the References[2-5]. The relativistic generalization of Levinson's theorem has also been well established[6-8]. However, most of these authors deal with the problem in ordinary three-dimensional space. To our knowledge a two-dimensional version of Levinson's theorem was not presented in the literature. The purpose of this work is to develop an analogue of this theorem in two spatial dimensions. It relates the phase shift at threshold for the $m$ th partial wave, $\eta_{m}(0)$, to the total number of bound states with angular momentum $m \hbar, n_{m}$ (the total number of bound states with angular momentum $-m \hbar$ is also $\left.n_{m}\right)$ :

$$
\eta_{m}(0)=n_{m} \pi, \quad m=0,1,2, \ldots
$$

This is similar to but slightly simpler than the original one in three dimensions. In three dimensions Levinson's theorem takes the same form with $m$ replaced by $l$. But when $l=0$ the relation must be modified if there exists a zero-energy resonance(a half-bound state). The mathematical origin is that the behaviour of the phase shifts $\delta_{l}(k)$ near $k=0$ may be different for $l=0$ and $l \neq 0$. In two dimensions no similar situation occurs. This paper is organized as follows. In the next section we give a brief formulation of the partial-wave method for nonrelativistic scattering in two spatial dimensions. In $\S 3$ we discuss the behaviour of the phase shifts near $k=0$ in some detail. In $\S 4$ we establish the Levinson theorem using the Green function method[2,3,5]. $\S 5$ is devoted to the discussion 
of some aspects of the theorem.

\section{$\S 2$. Partial-wave method in two dimensions}

A particle with mass $\mu$ and energy $E$ moving in an external field $V(\mathbf{r})$ satisfies the stationary Schrödinger equation

$$
H \psi=-\frac{\hbar^{2}}{2 \mu} \nabla^{2} \psi+V(\mathbf{r}) \psi=E \psi
$$

We use the polar coordinates $(r, \theta)$ as well as the rectangular coordinates $(x, y)$ in two spatial dimensions. For scattering problems $E>0$ (we assume that $V \rightarrow 0$ more rapidly than $r^{-2}$ when $\left.r \rightarrow \infty\right)$. The incident wave may be chosen as

$$
\psi_{i}=e^{i k x}
$$

which solves Eq.(1) when $r \rightarrow \infty$ provided that $k=\sqrt{2 \mu E / \hbar^{2}}$. The scattered wave should have the asymptotic form

$$
\psi_{s} \stackrel{r \rightarrow \infty}{\longrightarrow} \sqrt{\frac{i}{r}} f(\theta) e^{i k r}
$$

where the factor $\sqrt{i}=e^{i \pi / 4}$ is introduced for later convenience. This also solves (1) when $r \rightarrow \infty$. It is easy to show that the differential cross section $\sigma(\theta)$ (in two spatial dimensions the cross section may be more appropriately called cross width) is given in terms of the scattering amplitude $f(\theta)$ by

$$
\sigma(\theta)=|f(\theta)|^{2}
$$

The outgoing wave comprising (2) and (3) and thus takes the following form at infinity.

$$
\psi \stackrel{r \rightarrow \infty}{\longrightarrow} e^{i k x}+\sqrt{\frac{i}{r}} f(\theta) e^{i k r} .
$$

We are interested in central or spherically symmetric (actually cylindrically symmetric in two dimensions) potentials $V(\mathbf{r})=V(r)$. In this paper we deal only with central 
potentials. Then solutions of Eq.(1) may be expanded as

$$
\psi(r, \theta)=\sum_{m=-\infty}^{+\infty} a_{m} R_{|m|}(r) e^{i m \theta}
$$

where $R_{m}(r)$ satisfies the radial equation

$$
R_{m}^{\prime \prime}+\frac{1}{r} R_{m}^{\prime}+\left(k^{2}-\frac{2 \mu}{\hbar^{2}} V-\frac{m^{2}}{r^{2}}\right) R_{m}=0, \quad m=0,1,2, \ldots
$$

If $V=0$, the regular solution of $(7)$ is the Bessel function and may be taken as $R_{m}^{(0)}(r)=$ $\sqrt{k} J_{m}(k r)$ and hence has the asymptotic form

$$
R_{m}^{(0)}(r) \stackrel{r \rightarrow \infty}{\longrightarrow} \sqrt{\frac{2}{\pi r}} \cos \left(k r-\frac{m \pi}{2}-\frac{\pi}{4}\right) .
$$

We have assumed that $V(r) \rightarrow 0$ more rapidly than $r^{-2}$ when $r \rightarrow \infty$, so the solution $R_{m}(r)$ approaches the form of a linear combination of the Bessel function and the Neumann function at large $r$ and may have the asymptotic form

$$
R_{m}(r) \stackrel{r \rightarrow \infty}{\longrightarrow} \sqrt{\frac{2}{\pi r}} \cos \left[k r-\frac{m \pi}{2}-\frac{\pi}{4}+\eta_{m}(k)\right] .
$$

Here $\eta_{m}(k)$ is the phase shift of the $m$ th partial wave. It is a function of $k$. As in three dimensions, all $\eta_{m}(k)$ are real in a real potential. Substituting (9) into (6) gives one asymptotic form for $\psi$, while substitution of the formula

$$
e^{i k x}=\sum_{m=-\infty}^{+\infty} i^{|m|} J_{|m|}(k r) e^{i m \theta}
$$

and the asymptotic form of the Bessel functions into (5) gives another. Comparing these two asymptotic forms one finds an expression for $f(\theta)$ in terms of the phase shifts:

$$
f(\theta)=\sum_{m=-\infty}^{+\infty} \sqrt{\frac{2}{\pi k}} e^{i \eta_{|m|}} \sin \eta_{|m|} e^{i m \theta}
$$

The total cross section $\sigma_{t}$ turns out to be

$$
\sigma_{t}=\int_{0}^{2 \pi} d \theta \sigma(\theta)=\frac{4}{k}\left(\sin ^{2} \eta_{0}+2 \sum_{m=1}^{\infty} \sin ^{2} \eta_{m}\right) .
$$


From the above relations one easily realizes that all information of the scattering process is contained in the phase shifts $\eta_{m}(k)$. The latter are determined by solving the radial equation (7) with the boundary condition (9) and thus depend on the particular form of $V(r)$. In an attractive field, the number of bound states with given angular momentum $m \hbar$, denoted by $n_{m}$ above, also depends on the particular form of $V(r)$. It will be shown that $n_{m}$ is related to $\eta_{m}(k)$ at threshold. This is similar to Levinson's theorem in three dimensions. In the next section we first discuss the behaviour of $\eta_{m}(k)$ near $k=0$.

\section{$\S 3$. Phase shifts near threshold}

Assuming that $V(r)$ is less singular than $r^{-2}$ when $r \rightarrow 0$, then the regular solution of the radial equation (7) may have the following power dependence on $r$ near $r=0$.

$$
f_{m}(r, k) \stackrel{r \rightarrow 0}{\longrightarrow} \frac{r^{m}}{2^{m} m !}, \quad m=0,1,2, \ldots
$$

Here we denote the regular solution of (7) with the boundary condition (13) by $f_{m}(r, k)$. Note that the equation (7) depends on $k$ only through $k^{2}$, which is an integral function of $k$, and the boundary condition (13) is independent of $k$. Thus a theorem of Poincaré tells us that $f_{m}(r, k)$ is an integral function of $k$ for a fixed $r$. On the other hand, the solution $R_{m}(r)$ with the boundary condition (9), which is proportional to $f_{m}(r, k)$, need not be an integral function of $k$. We denote a potential that satisfies $V(r)=0$ when $r>a>0$ by $V_{a}(r)$. In such potentials the solution of (7) when $r>a$ may take the form

$$
R_{m}^{+}(r)=\sqrt{k}\left[\cos \eta_{m} J_{m}(k r)-\sin \eta_{m} N_{m}(k r)\right], \quad m=0,1,2, \ldots
$$

where the superscript "+" indicates $r>a$. It is easy to verify that $R_{m}^{+}(r)$ indeed satisfies the boundary condition (9). When $r<a$ we have

$$
R_{m}^{-}(r)=A_{m}(k) f_{m}(r, k), \quad m=0,1,2, \ldots
$$


where the superscript "-" indicates $r<a$. In general the coefficient $A_{m}$ depends on $k$, so that the two parts of $R_{m}(r)$ can be connected smoothly at $r=a$. This leads to

$$
\tan \eta_{m}=\frac{\rho J_{m}^{\prime}(\rho)-\beta_{m}(\rho) J_{m}(\rho)}{\rho N_{m}^{\prime}(\rho)-\beta_{m}(\rho) N_{m}(\rho)}
$$

where $\rho=k a$ and

$$
\beta_{m}(\rho)=\frac{a f_{m}^{\prime}(a, k)}{f_{m}(a, k)}
$$

where the prime indicates differentiation with respect to $r$. As mentioned above, $f_{m}(a, k)$ is an integral function of $k$, so is $f_{m}^{\prime}(a, k)$. Moreover, both of them are even functions of $k$ since Eq.(7) depends only on $k^{2}$. Therefore when $k \rightarrow 0$ or $\rho \rightarrow 0$, the leading term for $\beta_{m}(\rho)$ may have one of the following forms

$$
\begin{gathered}
\beta_{m}(\rho) \rightarrow \alpha_{m}^{+} \rho^{2 l_{m}^{+}}, \\
\beta_{m}(\rho) \rightarrow \alpha_{m}^{-} \rho^{-2 l_{m}^{-}}, \\
\beta_{m}(\rho) \rightarrow \gamma_{m}+\alpha_{m} \rho^{2 l_{m}}
\end{gathered}
$$

where $\alpha_{m}^{ \pm}, \alpha_{m}$, and $\gamma_{m}$ are nonzero constants, while $l_{m}^{ \pm}$and $l_{m}$ are natural numbers. Using these relations and the leading terms of $J_{m}(\rho)$ and $N_{m}(\rho)$ for $\rho \rightarrow 0$, the leading term in $\tan \eta_{m}$ when $\rho \rightarrow 0$ can be explicitly worked out. When $\gamma_{m}=-m$ some care should be taken. However, careful analysis gives in any case

$$
\tan \eta_{m} \rightarrow b_{m} \rho^{2 p_{m}} \quad \text { or } \quad \frac{\pi}{2 \ln \rho} \quad(k \rightarrow 0)
$$

where $b_{m} \neq 0$ is a contant and $p_{m}$ is a natural number. Eq.(18) is important for the development of the Levinson theorem in the next section.

For comparison we give the corresponding results in three dimensions. The phase shifts are denoted by $\delta_{l}(k)$. By similar analysis it can be shown in a potential $V_{a}(r)$ that

$$
\tan \delta_{l} \rightarrow c_{l} \rho^{2 q_{l}-1}, \quad l=0,1,2, \ldots \quad(k \rightarrow 0)
$$


where $c_{l} \neq 0$ is a constant, and $q_{l}$ is a natural number for $l \neq 0$, while $q_{0}$ may be a natural number or zero. We see that $\delta_{l}(0)$ generally equals a multiple of $\pi$ for all $l$. But $\delta_{0}(0)$ gets an additional $\pi / 2$ when $q_{0}=0$. The latter case does not occur for any $\eta_{m}(0)$, which is obvious from (18). The difference between (18) and $\left(18^{\prime}\right)$ comes from the fact that the Neumann function in the solution (14) involves the logarithmic function while the spherical Neumann function in the three-dimensional solution does not. It can be shown that $\delta_{0}(0)$ gets an additional $\pi / 2$ when there exists a half-bound state(a zero-energy resonance) in the angular momentum channel $l=0$.

\section{$\S 4$. The Levinson theorem}

Now we proceed to establish the Levinson theorem by the Green function method. Introduce the retarded Green function $G\left(\mathbf{r}, \mathbf{r}^{\prime}, E\right)$ defined by

$$
G\left(\mathbf{r}, \mathbf{r}^{\prime}, E\right)=\sum_{\nu} \frac{\psi_{\nu}(\mathbf{r}) \psi_{\nu}^{*}\left(\mathbf{r}^{\prime}\right)}{E-E_{\nu}+i \epsilon}
$$

where $\left\{\psi_{\nu}(\mathbf{r})\right\}$ is a complete set of orthonormal solutions to (1), and $\epsilon=0^{+} . G\left(\mathbf{r}, \mathbf{r}^{\prime}, E\right)$ satisfies the equation

$$
(E-H+i \epsilon) G\left(\mathbf{r}, \mathbf{r}^{\prime}, E\right)=\delta\left(\mathbf{r}-\mathbf{r}^{\prime}\right)
$$

For a free particle we have a similar definition:

$$
G^{(0)}\left(\mathbf{r}, \mathbf{r}^{\prime}, E\right)=\sum_{\nu} \frac{\psi_{\nu}^{(0)}(\mathbf{r}) \psi_{\nu}^{(0) *}\left(\mathbf{r}^{\prime}\right)}{E-E_{\nu}^{(0)}+i \epsilon}
$$

where $\left\{\psi_{\nu}^{(0)}(\mathbf{r})\right\}$ is a complete set of orthonormal solutions to Eq.(1) with $V=0 . G^{(0)}\left(\mathbf{r}, \mathbf{r}^{\prime}, E\right)$ satisfies

$$
\left(E-H_{0}+i \epsilon\right) G^{(0)}\left(\mathbf{r}, \mathbf{r}^{\prime}, E\right)=\delta\left(\mathbf{r}-\mathbf{r}^{\prime}\right)
$$


where $H_{0}$ is the Hamiltonian of the free particle. We have the integral equation for $G\left(\mathbf{r}, \mathbf{r}^{\prime}, E\right)$

$$
G\left(\mathbf{r}, \mathbf{r}^{\prime}, E\right)-G^{(0)}\left(\mathbf{r}, \mathbf{r}^{\prime}, E\right)=\int d \mathbf{r}^{\prime \prime} G^{(0)}\left(\mathbf{r}, \mathbf{r}^{\prime \prime}, E\right) V\left(\mathbf{r}^{\prime \prime}\right) G\left(\mathbf{r}^{\prime \prime}, \mathbf{r}^{\prime}, E\right)
$$

In a central field $V(\mathbf{r})=V(r)$ (not necessarily $\left.V_{a}(r)\right)$, we have

$$
\psi_{\nu}(r, \theta)=\psi_{m \kappa}(r, \theta)=\frac{u_{|m| \kappa}(r)}{\sqrt{r}} \frac{e^{i m \theta}}{\sqrt{2 \pi}}, \quad m=0, \pm 1, \pm 2, \ldots
$$

where $\kappa$ is a quantum number associated with the energy $E_{m \kappa}$ which is determined by solving the radial equation

$$
u_{m \kappa}^{\prime \prime}+\left[\frac{2 \mu}{\hbar^{2}}\left(E_{m \kappa}-V\right)-\frac{m^{2}-1 / 4}{r^{2}}\right] u_{m \kappa}=0, \quad m=0,1,2, \ldots
$$

with appropriate boundary conditions in the radial direction. The radial wave functions $u_{m \kappa}(r)$ satisfy the orthonormal condition

$$
\left(u_{m \kappa}, u_{m \kappa^{\prime}}\right)=\int_{0}^{\infty} d r u_{m \kappa}^{*}(r) u_{m \kappa^{\prime}}(r)=\delta_{\kappa \kappa^{\prime}}
$$

For an attractive field we have discrete $\operatorname{spectrum}\left(E_{m \kappa}<0\right)$ as well as continuous spectrum $\left(E_{m \kappa}>0\right)$. We can, however, require the wave functions to vanish at a sufficiently large radius $R\left(R \gg a\right.$ for $\left.V_{a}(r)\right)$ and thus discretize the continuous part of the spectrum. In this case the upper limit of the integration in (25) should be replaced by $R$. It is easy to show that

$$
G\left(\mathbf{r}, \mathbf{r}^{\prime}, E\right)=G_{0}\left(r, r^{\prime}, E\right) \frac{1}{2 \pi}+\sum_{m=1}^{\infty} G_{m}\left(r, r^{\prime}, E\right) \frac{\cos m\left(\theta-\theta^{\prime}\right)}{\pi}
$$

where

$$
G_{m}\left(r, r^{\prime}, E\right)=\sum_{\kappa} \frac{u_{m \kappa}(r) u_{m \kappa}^{*}\left(r^{\prime}\right)}{\sqrt{r r^{\prime}}\left(E-E_{m \kappa}+i \epsilon\right)}, \quad m=0,1,2, \ldots
$$

For a free particle, the following results can be obtained in the same way.

$$
G^{(0)}\left(\mathbf{r}, \mathbf{r}^{\prime}, E\right)=G_{0}^{(0)}\left(r, r^{\prime}, E\right) \frac{1}{2 \pi}+\sum_{m=1}^{\infty} G_{m}^{(0)}\left(r, r^{\prime}, E\right) \frac{\cos m\left(\theta-\theta^{\prime}\right)}{\pi}
$$


where

$$
G_{m}^{(0)}\left(r, r^{\prime}, E\right)=\sum_{\kappa} \frac{u_{m \kappa}^{(0)}(r) u_{m \kappa}^{(0) *}\left(r^{\prime}\right)}{\sqrt{r r^{\prime}}\left(E-E_{m \kappa}^{(0)}+i \epsilon\right)}, \quad m=0,1,2, \ldots
$$

where $u_{m \kappa}^{(0)}(r)$ satisfies (24) with $V=0$, and the energy $\operatorname{spectrum}\left(E_{m \kappa}^{(0)}>0\right)$ is discretized according to the above described prescription. Thus the orthonormal relation for $u_{m \kappa}^{(0)}(r)$ is similar to (25). Substituting (26) and (28) into (23) we get an integral equation for $G_{m}\left(r, r^{\prime}, E\right)$

$G_{m}\left(r, r^{\prime}, E\right)-G_{m}^{(0)}\left(r, r^{\prime}, E\right)=\int d r^{\prime \prime} r^{\prime \prime} G_{m}^{(0)}\left(r, r^{\prime \prime}, E\right) V\left(r^{\prime \prime}\right) G_{m}\left(r^{\prime \prime}, r^{\prime}, E\right), \quad m=0,1,2, \ldots$

Using the orthonormal relation (25) it is easy to show that

$$
\int d r r G_{m}(r, r, E)=\sum_{\kappa} \frac{1}{E-E_{m \kappa}+i \epsilon} .
$$

Employing the mathematical formula

$$
\frac{1}{x+i \epsilon}=P \frac{1}{x}-i \pi \delta(x)
$$

and taking the imaginary part of the above equation we have

$$
\operatorname{Im} \int d r r G_{m}(r, r, E)=-\pi \sum_{\kappa} \delta\left(E-E_{m \kappa}\right)
$$

Integrating this equation over $E$ from $-\infty$ to $0^{-}$yields

$$
\operatorname{Im} \int_{-\infty}^{0^{-}} d E \int d r r G_{m}(r, r, E)=-n_{m}^{-} \pi
$$

where $n_{m}^{-}$is the number of bound states with negative energies and with angular momentum $m \hbar$ (when $m \neq 0$ we have the same number of bound states with angular momentum $-m \hbar$ as well). The possibility of a zero-energy bound state will be discussed in the next section. In a similar way one can show that

$$
\operatorname{Im} \int_{-\infty}^{0^{-}} d E \int d r r G_{m}^{(0)}(r, r, E)=0
$$


Here and in (34) the integration over $E$ is performed to the upper limit $0^{-}$instead of 0 such that it suffers no ambiguity. Combining (34) and (35) we have

$$
\operatorname{Im} \int_{-\infty}^{0^{-}} d E \int d r r\left[G_{m}(r, r, E)-G_{m}^{(0)}(r, r, E)\right]=-n_{m}^{-} \pi
$$

On the other hand, substituting (27) and (29) into the rhs of (30) we have

$$
\int d r r\left[G_{m}(r, r, E)-G_{m}^{(0)}(r, r, E)\right]=\sum_{\kappa \sigma} \frac{\left(u_{m \sigma}, u_{m \kappa}^{(0)}\right)\left(u_{m \kappa}^{(0)}, V u_{m \sigma}\right)}{\left(E-E_{m \kappa}^{(0)}+i \epsilon\right)\left(E-E_{m \sigma}+i \epsilon\right)} .
$$

Using the radial equations for $u_{m \kappa}^{(0)}(r)$ and $u_{m \sigma}(r)$ and the boundary condition that these radial wave functions vanish at $r=0$ and $r=R$, it is not difficult to show that

$$
\left(u_{m \kappa}^{(0)}, V u_{m \sigma}\right)=\left(E_{m \sigma}-E_{m \kappa}^{(0)}\right)\left(u_{m \kappa}^{(0)}, u_{m \sigma}\right) .
$$

Substituting this result into (37) and taking the imaginary part we have

$$
\operatorname{Im} \int d r r\left[G_{m}(r, r, E)-G_{m}^{(0)}(r, r, E)\right]=\pi \sum_{\kappa \sigma}\left[\delta\left(E-E_{m \kappa}^{(0)}\right)-\delta\left(E-E_{m \sigma}\right)\right]\left|\left(u_{m \kappa}^{(0)}, u_{m \sigma}\right)\right|^{2}
$$

Integrating this equation over $E$ from $-\infty$ to $+\infty$ it is easy to find that

$$
\operatorname{Im} \int_{-\infty}^{+\infty} d E \int d r r\left[G_{m}(r, r, E)-G_{m}^{(0)}(r, r, E)\right]=0
$$

Similar to the three-dimensional case, this equation means that the total number of states in a specific angular momentum channel is not altered by an attractive field, except that some scattering states are pulled down into the bound-state region. This result, together with (36), leads to

$$
\operatorname{Im} \int_{0^{-}}^{+\infty} d E \int d r r\left[G_{m}(r, r, E)-G_{m}^{(0)}(r, r, E)\right]=n_{m}^{-} \pi
$$

We have thereupon finished the first step in our establishment of the Levinson theorem.

The next step is to calculate the lhs of (41) in another way. In the above treatment we have discretized the continuous spectrum of $E_{m \kappa}^{(0)}$ and the continuous part of $E_{m \kappa}$. In 
the following we will directly deal with these continuous spectra. We will denote $u_{m \kappa}^{(0)}(r)$ by $u_{m k}^{(0)}(r)$, and $u_{m \kappa}(r)$ with continuous $\kappa$ by $u_{m k}(r)$, whereas those $u_{m \kappa}(r)$ with discrete $\kappa$ (bound states) will be denoted by the original notation. The notations $E_{m \kappa}^{(0)}$ and $E_{m \kappa}$ with continuous $\kappa$ will also be changed to $E_{m k}^{(0)}$ and $E_{m k}$ (both are equal to $\left.\hbar^{2} k^{2} / 2 \mu\right)$ respectively. The orthonormal relation for $u_{m k}^{(0)}(r)$ now takes the form

$$
\left(u_{m k}^{(0)}, u_{m k^{\prime}}^{(0)}\right)=\delta\left(k-k^{\prime}\right)
$$

The orthonormal relation for $u_{m k}(r)$ is similar, while that for $u_{m \kappa}(r)$ has the same appearance as (25). It is easy to show that

$$
u_{m k}^{(0)}(r)=\sqrt{k r} J_{m}(k r), \quad k=\sqrt{2 \mu E_{m k}^{(0)}} / \hbar
$$

satisfies the radial equation with $V=0$ and the orthonormal relation (42). Thus $u_{m k}^{(0)}(r)$ has the asymptotic form

$$
u_{m k}^{(0)}(r) \stackrel{r \rightarrow \infty}{\longrightarrow} \sqrt{\frac{2}{\pi}} \cos \left(k r-\frac{m \pi}{2}-\frac{\pi}{4}\right)
$$

corresponding to (8). In an external field, the wave functions are distorted and thus the asymptotic form for $u_{m k}(r)$ becomes

$$
u_{m k}(r) \stackrel{r \rightarrow \infty}{\longrightarrow} \sqrt{\frac{2}{\pi}} \cos \left[k r-\frac{m \pi}{2}-\frac{\pi}{4}+\eta_{m}(k)\right]
$$

corresponding to (9). Note that the coefficient in the asymptotic form is the same for $u_{m k}^{(0)}(r)$ and $u_{m k}(r)$. In this treatment it can be shown that

$$
G_{m}\left(r, r^{\prime}, E\right)=\sum_{\kappa} \frac{u_{m \kappa}(r) u_{m \kappa}^{*}\left(r^{\prime}\right)}{\sqrt{r r^{\prime}}\left(E-E_{m \kappa}+i \epsilon\right)}+\int d k \frac{u_{m k}(r) u_{m k}^{*}\left(r^{\prime}\right)}{\sqrt{r r^{\prime}}\left(E-E_{m k}+i \epsilon\right)}
$$

and thus

$$
\operatorname{Im} \int d r r G_{m}(r, r, E)=-\pi \sum_{\kappa} \delta\left(E-E_{m \kappa}\right)-\pi \int d k \delta\left(E-E_{m k}\right)\left(u_{m k}, u_{m k}\right)
$$


Integrating this equation over $E$ from $0^{-}$to $+\infty$ and taking into account the fact that $E_{m \kappa}<0$ while $E_{m k} \geq 0$, we have

$$
\operatorname{Im} \int_{0^{-}}^{+\infty} d E \int d r r G_{m}(r, r, E)=-\pi \int d k\left(u_{m k}, u_{m k}\right)
$$

There is no ambiguity in the integration over $E$ since the lower limit is set to be $0^{-}$instead of 0 (note that $E_{m k}$ may equal 0 ). As before, the possibility of a zero-energy bound state will be discussed in $§ 5$. In the same way, we have

$$
\operatorname{Im} \int_{0^{-}}^{+\infty} d E \int d r r G_{m}^{(0)}(r, r, E)=-\pi \int d k\left(u_{m k}^{(0)}, u_{m k}^{(0)}\right)
$$

It should be pointed out that the integrands in both (47) and (48) are $\delta(0)(=\infty)$ according to Eq.(42) and a similar equation for $u_{m k}(r)$. However, there is a subtle difference between these two $\delta$ functions, and it is this difference that leads to the Levinson theorem. Since both integrands are singular, we first evaluate

$$
\left(u_{m k}, u_{m l}\right)_{r_{0}}-\left(u_{m k}^{(0)}, u_{m l}^{(0)}\right)_{r_{0}} \equiv \int_{0}^{r_{0}} d r u_{m k}^{*}(r) u_{m l}(r)-\int_{0}^{r_{0}} d r u_{m k}^{(0) *}(r) u_{m l}^{(0)}(r)
$$

where $r_{0}$ is a large but finite radius, and finally take the limit $l \rightarrow k$ and $r_{0} \rightarrow \infty$. As in the discretized case, we have the boundary condition

$$
u_{m k}^{(0)}(0)=0, \quad u_{m k}(0)=0
$$

Using the radial equation and this boundary condition it is easy to show that

$$
\left(k^{2}-l^{2}\right)\left(u_{m k}, u_{m l}\right)_{r_{0}}=u_{m k}^{*}\left(r_{0}\right) u_{m l}^{\prime}\left(r_{0}\right)-u_{m k}^{\prime *}\left(r_{0}\right) u_{m l}\left(r_{0}\right)
$$

Since $r_{0}$ is large, we can use (44) to evaluate the rhs and in the limit $l \rightarrow k$ we get

$$
\left(u_{m k}, u_{m k}\right)_{r_{0}}=\frac{r_{0}}{\pi}+\frac{1}{\pi} \eta_{m}^{\prime}(k)-\frac{(-)^{m}}{2 \pi k} \cos \left[2 k r_{0}+2 \eta_{m}(k)\right] .
$$


In the same way we have

$$
\left(u_{m k}^{(0)}, u_{m k}^{(0)}\right)_{r_{0}}=\frac{r_{0}}{\pi}-\frac{(-)^{m}}{2 \pi k} \cos 2 k r_{0} .
$$

Therefore,

$$
\left(u_{m k}, u_{m k}\right)_{r_{0}}-\left(u_{m k}^{(0)}, u_{m k}^{(0)}\right)_{r_{0}}=\frac{1}{\pi} \eta_{m}^{\prime}(k)+\frac{(-)^{m}}{2} \delta(k) \sin 2 \eta_{m}(k)+\frac{(-)^{m}}{\pi k} \cos 2 k r_{0} \sin ^{2} \eta_{m}(k)
$$

where we have employed the well-known formula

$$
\lim _{r_{0} \rightarrow \infty} \frac{\sin 2 k r_{0}}{\pi k}=\delta(k)
$$

So far in this section $V(r)$ need not be $V_{a}(r)$. In the following we set $V(r)=V_{a}(r)$. Then Eq.(18) is valid, and we have $\sin 2 \eta_{m}(0)=0$. Therefore the second term on the rhs of (54) vanishes. Integrating this result over $k$ (from 0 to $+\infty$ ), taking the limit $r_{0} \rightarrow \infty$, and incorporating the results (47), (48) we arrive at

$$
\begin{aligned}
& \operatorname{Im} \int_{0^{-}}^{+\infty} d E \int d r r\left[G_{m}(r, r, E)-G_{m}^{(0)}(r, r, E)\right] \\
& =\eta_{m}(0)-\eta_{m}(\infty)-(-)^{m} \lim _{r_{0} \rightarrow \infty} \int_{0}^{\infty} d k \frac{\cos 2 k r_{0}}{k} \sin ^{2} \eta_{m}(k) .
\end{aligned}
$$

The last term in this equation can be decomposed into two integrals, the first from 0 to $\varepsilon=0^{+}$, which can be shown to vanish on account of (18), while the second from $\varepsilon$ to $+\infty$, which also vanishes in the limit $r_{0} \rightarrow \infty$ since the factor $\cos 2 k r_{0}$ oscillates very rapidly. Therefore we have

$$
\operatorname{Im} \int_{0^{-}}^{+\infty} d E \int d r r\left[G_{m}(r, r, E)-G_{m}^{(0)}(r, r, E)\right]=\eta_{m}(0)-\eta_{m}(\infty)
$$

Combining (56) and (41) we arrive at the Levinson theorem:

$$
\eta_{m}(0)-\eta_{m}(\infty)=n_{m}^{-} \pi, \quad m=0,1,2, \ldots
$$


In the next section we will discuss some aspects of this theorem, and get a more general form which takes zero-energy bound states into consideration.

\section{$\S 5$. Discussions}

In this section we discuss and clarify some aspects of the Levinson theorem obtained in the form (57) in the last section.

1. On zero-energy bound states. In $\S 4$ we have not taken into account the possible existence of a zero-energy bound state. Indeed, this may occur for a square well potential with radius $a$ and depth $V_{0}$ when $m>1$ and $J_{m-1}(\xi)=0$ where $\xi=k_{0} a$ and $k_{0}=$ $\sqrt{2 \mu V_{0}} / \hbar$. (For $m=0,1$ regular solutions with zero energy may be found but they are not normalizable and thus are not bound states. see below) The existence of a zero-energy bound state would not alter the results (34)-(36) where $n_{m}^{-}$is the number of bound states

with negative energies. Therefore Eq.(41) remains valid in this case. On the other hand, Eq.(47) becomes

$$
\operatorname{Im} \int_{0^{-}}^{+\infty} d E \int d r r G_{m}(r, r, E)=-\pi-\pi \int d k\left(u_{m k}, u_{m k}\right)
$$

which is an obvious consequence of (46). Accordingly, the result (56) becomes

$$
\operatorname{Im} \int_{0^{-}}^{+\infty} d E \int d r r\left[G_{m}(r, r, E)-G_{m}^{(0)}(r, r, E)\right]=\eta_{m}(0)-\eta_{m}(\infty)-\pi
$$

Hence, the Levinson theorem takes in the present case the form

$$
\eta_{m}(0)-\eta_{m}(\infty)=n_{m} \pi
$$

where $n_{m}=n_{m}^{-}+1$ represents the total number of bound states, including the one with zero energy. When there is no zero-energy bound state, $n_{m}=n_{m}^{-}$. Thus Eq.(60) holds in any case and is the final form of the Levinson theorem. 
To see the difference in zero-energy states between two and three dimensions, we notice that the two-dimensional radial wave function $u_{m \kappa}(r)$ satisfies $(24)$. When regarded as a one-dimensional Schrödinger equation, the effective potential reads

$$
\tilde{V}_{2}(r)=V(r)+\frac{\hbar^{2}\left(m^{2}-1 / 4\right)}{2 \mu r^{2}}
$$

where the subscript "2" indicates two dimensions. In three dimensions, with $\psi_{l m \kappa}(r, \theta, \phi)=$ $r^{-1} \chi_{l \kappa}(r) Y_{l m}(\theta, \phi)$ (here $r, \theta$ etc. should not be confused with those in two dimensions), the radial wave function $\chi_{l \kappa}(r)$ satisfies

$$
\chi_{l \kappa}^{\prime \prime}+\left[\frac{2 \mu}{\hbar^{2}}\left(E_{l \kappa}-V\right)-\frac{l(l+1)}{r^{2}}\right] \chi_{l \kappa}=0, \quad l=0,1,2, \ldots
$$

When this is regarded as a one-dimensional Schrödinger equation, the effective potential is

$$
\tilde{V}_{3}(r)=V(r)+\frac{\hbar^{2} l(l+1)}{2 \mu r^{2}}
$$

which is obviously different from $\tilde{V}_{2}(r)$, and as a consequence the zero-energy solutions are different from those of (24). More specifically, with $V(r)=V_{a}(r)$ and $E_{m 0}=0$, the exterior solution $(r>a)$ of $(24)$ reads

$$
u_{m 0}^{+}(r)=r^{-m+1 / 2}
$$

A zero-energy solution exists if $V_{a}(r)$ is such that the interior solution $u_{m 0}^{-}(r)$ can be connected with $u_{m 0}^{+}(r)$ at $r=a$ smoothly. This leads to the above mentioned condition for a square well potential. Here we are concerned about the normalizability of the solution. It is clear that the above solution can be normalized and thus is a bound state only when $m>1$. We have $\psi_{10} \stackrel{r \rightarrow \infty}{\longrightarrow} 0$, and $\psi_{00}$ is finite at infinity though $u_{00}(r) \stackrel{r \rightarrow \infty}{\longrightarrow} \infty$, thus both are regular. But either of them can not be normalized and thus is not a bound state. 
In this case $\eta_{m}(0)$ gets no additional term and Eq.(57) is not modified. When $m>1$, however, $n_{m}^{-} \rightarrow n_{m}^{-}+1=n_{m}$ and $\eta_{m}(0)$ gets an additional $\pi$ if a zero-energy solution(a bound state) actually exists. In three dimensions, with $V(r)=V_{a}(r)$ and $E_{l 0}=0$, the exterior solution of $\left(24^{\prime}\right)$ reads

$$
\chi_{l 0}^{+}(r)=r^{-l}
$$

This is normalizable when $l>0$. Thus only $l=0$ is distinguished. When the $l=0$ solution really emerges, $\delta_{0}(0)$ gets an additional $\pi / 2$ as pointed out in $\S 3$, and the Levinson theorem is modified by $n_{0} \rightarrow n_{0}+1 / 2$. This is different from the case $m=0,1$ in two dimensions where $\eta_{m}(0)$ always equals a multiple of $\pi$. When $l>0$ the case is basically the same as $m>1$ in two dimensions.

2. About $\eta_{m}(\infty)$. Write down two radial equations for $u_{m k}(r)$ and $\tilde{u}_{m k}(r)$ in the external potentials $U(r)$ and $\tilde{U}(r)$ respectively. Using the boundary condition (50) and the asymptotic form (44) for $u_{m k}(r)$ and similar ones for $\tilde{u}_{m k}(r)$, it is easy to show that

$$
\sin \left[\eta_{m}(k)-\tilde{\eta}_{m}(k)\right]=-\frac{\pi \mu}{\hbar^{2} k} \int_{0}^{\infty} d r[U(r)-\tilde{U}(r)] u_{m k}^{*}(r) \tilde{u}_{m k}(r)
$$

Now we take $U(r)=V(r)$ (not necessarily be $\left.V_{a}(r)\right)$ and $\tilde{U}(r)=0$, it is natural to define $\tilde{\eta}_{m}(k)=0$ in the absence of an external field. Obviously, $\tilde{u}_{m k}(r)=u_{m k}^{(0)}(r)$, so $(61)$ becomes

$$
\sin \eta_{m}(k)=-\frac{\pi \mu}{\hbar^{2} k} \int_{0}^{\infty} d r u_{m k}^{*}(r) V(r) u_{m k}^{(0)}(r) .
$$

For very large $k, V(r)$ can be ignored in the radial equation since it is less singular than $r^{-2}$ near the origin and well behaved elsewhere as assumed and so can be neglected everywhere(cf. Eq.(24)). Therefore $u_{m k}(r)$ in $(62)$ can be replaced by $u_{m k}^{(0)}(r)$ in this limit, and we have for very large $k$

$$
\sin \eta_{m}(k)=-\frac{\pi \mu}{\hbar^{2} k} \int_{0}^{\infty} d r\left|u_{m k}^{(0)}(r)\right|^{2} V(r) .
$$


Substituting $u_{m k}^{(0)}(r)=\sqrt{k r} J_{m}(k r)$ into this equation, and using the asymptotic formula for the Bessel functions at large argument, we have approximately

$$
\sin \eta_{m}(k)=-\frac{2 \mu}{\hbar^{2} k} \int_{0}^{\infty} d r \cos ^{2}\left(k r-\frac{m \pi}{2}-\frac{\pi}{4}\right) V(r) .
$$

Further, since $k$ is very large, the cosine oscillates very rapidly and thus the squared cosine may be replaced by its mean value $1 / 2$. In this way we arrive at

$$
\sin \eta_{m}(k)=-\frac{\mu}{\hbar^{2} k} \int_{0}^{\infty} d r V(r)
$$

for very large $k$, where we assume that the integral exists. This result has the same form as that in three dimensions. Of course it holds in the special case $V(r)=V_{a}(r)$. Obviously, $\sin \eta_{m}(k) \rightarrow 0$ when $k \rightarrow \infty$, hence we can freely define $\eta_{m}(\infty)=0$. Under this convention the Levinson theorem takes the form

$$
\eta_{m}(0)=n_{m} \pi, \quad m=0,1,2, \ldots
$$

This means that the phase shift at threshold serves as a counter for the bound states. This is similar to the case in three dimensions, but is somewhat simpler. In three dimensions, the case $l=0$ should be modified when there exists a zero-energy resonance(a half-bound state). Here we have no such problem.

3. Extension to more general potentials. In the above development of the Levinson theorem, we have assumed that $V(r)=0$ when $r>a$. We also assumed that $V(r)$ is less singular than $r^{-2}$ when $r \rightarrow 0$, so that the power dependence in (13) near $r=0$ is valid. Indeed, the existence of the integral in (65) requires that $V(r)$ is less singular than $r^{-1}$ when $r \rightarrow 0$. We will always assume that $V(r)$ is sufficiently well behaved near the origin such that the above requirements are all satisfied. On the other hand, the radius $a$ beyond which $V(r)$ vanishes is not specified in our discussion. Though both $\eta_{m}(0)$ and $n_{m}$ in (66) 
depend on the particular form of $V(r)$ and thus depend on $a$, the equality between them does not. Hence one expects that (66) remains valid when $V_{a}(r)$ is varied continuously to the limit $a \rightarrow \infty$ if $n_{m}$ remains finite in the process. It seems that the Levinson theorem holds for quite general potentials(well behaved near the origin as emphasized above) as long as they decrease rapidly enough when $r \rightarrow \infty$ such that the total number of bound states in a particular angular momentum channel is finite.

Extension of the present work to relativistic quantum mechanics is currently under progress.

The author is grateful to Prof. Guang-jiong Ni for useful communications and for encouragement. This work was supported by the National Natural Science Foundation of China. 


\section{References}

[1] N. Levinson, K. Dan. Vidensk. Selsk. Mat.-Fys. Medd. 25, No.9(1949).

[2] J. M. Jauch, Helv. Phys. Acta. 30, 143(1957).

[3] A. Martin, Nuovo Cimento 7, 607(1958).

[4] R. G. Newton, J. Math. Phys. 18, 1348; 1582(1977);

Scattering Theory of Waves and Particles(McGraw-Hill, New York, 1966).

[5] G.-J. Ni, Phys. Energ. Fort. Phys. Nucl. 3, 432(1979).

[6] Z.-Q. Ma and G.-J. Ni, Phys. Rev. D31, 1482(1985).

[7] N. Poliatzky, Phys. Rev. Lett. 70, 2507(1993); Helv. Phys. Acta. 66, 241(1993).

[8] J. Piekarewicz, Phys. Rev. C48, 2174(1993). 